\title{
Dor abdominal crônica em crianças e adolescentes: aspectos gerais
}

\author{
Chronic abdominal pain in children and adolescents: general aspects \\ Dolor abdominal crónico en niños y adolescentes: aspectos generales
}

Eliane Alves Motta Cabello dos Santos ${ }^{1 *}$, Thais Marchini de Oliveira ${ }^{1}$, Isabel Cristina Drago Salmen ${ }^{1}$.

\section{RESUMO}

Objetivo: Realizar uma revisão crítica da literatura sobre a Dor Abdominal Crônica (DAC) em crianças e adolescentes, com foco na dor de etiologia funcional. Revisão bibliográfica: A DAC tem alta prevalência em pediatria. O termo DAC inclui a dor de etiologia orgânica e a dor funcional. Na maioria dos casos, a DAC é considerada um distúrbio funcional do trato gastrintestinal, sendo definida como uma alteração do eixo cérebro intestinal. Segundo o Comitê de ROMA IV, a DAC funcional pode ser classificada em Dispepsia funcional, Síndrome do Intestino Irritável, Migrânea abdominal e Dor Abdominal Funcional sem outra especificação. O tratamento da dor funcional inclui educação, modificação dos fatores de estresse, intervenções dietéticas e quando necessárias, farmacoterapia e psicoterapia. Considerações finais: A DAC em pediatria representa um grande desafio. Constitui uma queixa de alta prevalência e que interfere no cotidiano do paciente. Recomenda-se uma abordagem biopsicossocial para evolução favorável. Muitos estudos ainda são necessários para otimizar compreensão e manejo do paciente pediátrico com DAC.

Palavras-chave: Dor abdominal, Crianças, Adolescentes.

\begin{abstract}
Objective: To review the current scientific literature on Chronic Abdominal Pain (CAP) in children and adolescents, with focus on the functional etiology. Bibliography review: CAP is highly prevalent in the general pediatric population. CAP is a descriptive term that includes organic and functional etiology for abdominal pain. Most CAP cases are functional conditions and have been defined as a disorder of the brain-gut axis. The ROME IV Committee has classified the Functional abdominal pain disorders as Functional Dyspepsia, Irritable Bowel Syndrome, Abdominal Migraine and Functional Abdominal Pain Not Otherwise Specified. The treatment includes education, modification of stress factors, dietary interventions and when necessary, pharmacotherapy and psychotherapy. Final considerations: The CAP represents a challenge in pediatrics. CAP has a high prevalence and may interferes with the patient's daily life. A biopsychosocial approach is recommended for a favorable outcome. Further studies are necessary for a better understanding and management of CAP in the pediatric population.
\end{abstract}

Keywords: Abdominal pain, Children, Adolescents.

\section{RESUMEN}

Objetivo: Revisar la literatura científica actual sobre el Dolor Abdominal Crónico (DAC), centrándose en la etiologíafuncional del dolor en niños y adolescentes. Revisión bibliográfica: El DAC en pediatría es una causa común de consulta médica. El término DAC incluye el dolor de etiología orgánica y funcional. En la mayoría de los casos, se considera el DAC un trastorno funcional del tracto gastrointestinal, definiéndose como una alteración del eje cerebral intestinal. Según los criterios de Roma IV para niños y adolescentes, se clasifica el DAC en: Dispepsia funcional, Síndrome del intestino irritable, Migraña abdominal, Dolor abdominal funcional sin otra especificación. El tratamiento del DAC funcional incluye educación, modificación de los factores de estrés, intervenciones dietéticas y, cuando sea necesario, farmacoterapia y psicoterapia. Consideraciones finales: EI DAC constituye un desafío constante para pediatras. Es una entidad clínica frecuente, que interfiere en la vida diaria del paciente. Se recomienda un abordaje biopsicosocial para un resultado favorable. Se necesitan más estudios para optimizar la comprensión y el tratamiento de los pacientes con DAC pediátrica.

Palabras clave: Dolor abdominal, Niños, Adolescentes.

${ }^{1}$ Hospital de Reabilitação de Anomalias Craniofaciais (HRAC) da Universidade de São Paulo (USP), Bauru SP. *E-mail: elianecabello@usp.br 


\section{INTRODUÇÃO}

A Dor Abdominal Crônica (DAC) representa um sintoma de alta prevalência em crianças e adolescentes, com média mundial estimada em 13,9\% (95\% IC 11.8-15.3) (KORTERINK JJ, et al., 2015). Manifesta-se geralmente com episódios recorrentes de evolução crônica e afeta profundamente o cotidiano do paciente (BRUSAFERRO A, et al., 2018). Acarretam impacto negativo na qualidade de vida, tornando-se um grande desafio para pais e pediatras (KLEINMAM RE, et al., 2018).

A DAC foi definida inicialmente em 1958 e descrita como uma síndrome dolorosa que acomete crianças com 3 anos ou mais, com pelo menos três episódios de dor abdominal, por pelo menos três meses durante 0 ano anterior e com episódios fortes o suficiente para interromper as atividades do paciente (APLEY J e NAISH $\mathrm{N}, 1958)$. Os critérios de APLEY para definição de DAC, permanece sendo de escolha para muitos estudos atuais (KORTERINK JJ, et al., 2015).

O termo DAC abrange a dor abdominal de etiologia orgânica e a dor abdominal funcional (DI LORENZO C, et al., 2005). A maioria dos casos de DAC é considerada como um Distúrbio Funcional do Trato Gastrointestinal (DFTGI), sendo entendida como um distúrbio do eixo cérebro intestinal. A DAC de etiologia funcional ocorre na ausência de fatores patológicos de base e tem sido estudada, classificada e seus critérios periodicamente divulgados pelo Comitê de ROMA desde 1988 (DROSSMAN DA, 2016).

O diagnóstico da DAC baseia-se na entrevista com os pais e com a criança e no exame físico, sendo de interesse a diferenciação entre etiologia orgânica ou funcional (NIGHTINGALE S e SHARMA A, 2020). No entanto, são poucos os itens da história e do exame físico que sinalizem a possibilidade de uma doença de base. De acordo com especialistas é a presença dos chamados sinais de alarme (SA) que sugere maior probabilidade de uma patologia como causa de DAC (Di LORENZO C, et al., 2005, HYAMS JS, et al., 2016).

Os SA clássicos incluem perda de peso inexplicável, presença de sangue nas fezes, desaceleração do crescimento físico, vômitos ou diarreias significantes, febre de origem indeterminada e disfagia (DI LORENZO C, et al., 2005; CHIOU E e NURKO S, 2011, REUST CE e WILLIAMS A, 2018).

O manejo do paciente com dor de etiologia orgânica é voltado para doença de base, enquanto os casos funcionais requerem tratamento complexo, com abordagem biopsicossocial e parceria médico-famíliapaciente (BRUSAFERRO A, et al., 2018). São componentes do tratamento da dor funcional: a educação do paciente e da família, a modificação dos fatores físicos e psicológicos de estresse, as intervenções dietéticas e quando necessárias, farmacoterapia e psicoterapia (DROSSMAN DA, 2016; NIGHTINGALE S e SHARMA A, 2020).

Ressalta-se que é improvável que qualquer terapia isoladamente obtenha a cura definitiva deste problema crônico. Muitos estudos são necessários para otimização do tratamento da DAC em pediatria e para melhor compreensão do tema (KLEINMAM RE, et al., 2018).

O objetivo desta pesquisa foi o de realizar uma revisão narrativa ampla do assunto DAC em crianças e adolescentes, abrangendo a epidemiologia, fisiopatologia e manejo do paciente.

\section{REVISÃO BIBLIOGRÁFICA}

\section{Epidemiologia da DAC}

A prevalência da DAC em pediatria é elevada, havendo uma ampla variação entre os trabalhos realizados nas diferentes regiões do mundo (KORTERINK JJ, et al, 2015). Esta divergência decorre dos múltiplos critérios de definição, das metodologias utilizadas e diferenças ambientais e genéticas. Entre crianças europeias, a prevalência de DAC é de cerca de 10\%, e no Brasil, segundo pesquisa realizada em duas cidades diferentes, encontrou-se prevalência de $21,6 \%$ e $21,9 \%$ (KORTERINK JJ, et al., 2015).

Meninas são mais acometidas por DAC quando comparadas aos meninos $(1,4: 1)$ na maioria dos estudos, em decorrência de questões hormonais ou maior tendência de verbalização da dor pelas meninas (KORTERINK JJ, et al., 2015). 


\section{Definição da Dor Abdominal Crônica}

Segundo a Sociedade Norte Americana de Gastroenterologia Pediátrica, Hepatologia e Nutrição (NASPGHAN) a DAC consiste em um quadro de dor abdominal de longa duração (usualmente mais de três meses) que pode se apresentar com um padrão contínuo ou intermitente (Dor Abdominal Recorrente ou DAR). Ressalta-se que os termos DAC ou DAR são descritivos e não diagnósticos, pois abrangem a dor abdominal de etiologia orgânica e a dor abdominal funcional (DI LORENZO C, et al., 2005).

\section{Etiologia e fisiopatologia: dor abdominal crônica de causa orgânica}

A fisiopatologia da DAC de etiologia orgânica está relacionada à lesão tissular inerente a cada tipo específico de doença, podendo ser associada principalmente à inflamação, a um processo obstrutivo, metabólico, neoplásico ou infeccioso (Quadro 1) (KLEINMAM RE, et al., 2018). Uma causa orgânica é encontrada na minoria das crianças com DAC, de 5 a 10\% dos casos (REUST CE e WILLIAMS A, 2018).

Quadro 1 - Causas principais de dor abdominal de etiologia orgânica.

\begin{tabular}{|c|c|}
\hline \multirow{8}{*}{ Trato gastrointestinal } & Refluxo gastroesofágico \\
\hline & Gastrite \\
\hline & Úlcera \\
\hline & Esofagite \\
\hline & Parasitoses \\
\hline & Doença Inflamatória Intestinal \\
\hline & Divertículo de Meckel \\
\hline & Má rotação com vólvulos intermitentes \\
\hline \multirow{5}{*}{ Trato urogenital } & Infecção urinária \\
\hline & Hidronefrose \\
\hline & Urolitíase \\
\hline & Dismenorreia \\
\hline & Doença Inflamatória pélvica \\
\hline \multirow{4}{*}{ Vesícula, fígado e pâncreas } & Cálculo vesical \\
\hline & Pancreatite \\
\hline & Abcesso hepático \\
\hline & Hepatite \\
\hline \multirow{4}{*}{ Miscelânia } & Neoplasias \\
\hline & Anemia falciforme \\
\hline & Vasculites \\
\hline & Porfiria \\
\hline
\end{tabular}

Fonte: Santos EAMC, et al., 2021; adaptado de Kleinmam RE, et al., 2018.

\section{Distúrbios da Dor Abdominal crônica de origem Funcional}

Os critérios de ROMA são diretrizes baseadas em sintomas clínicos, que têm possibilitado a padronização das definições dos DFTGI e os estudos da sua fisiopatologia e tratamento, sob diferentes pontos de vista (HYAMS JS, et al., 2016). São definidos os seguintes distúrbios da dor abdominal funcional para crianças e adolescentes de quatro a dezoito anos (critérios de ROMA IV): Dispepsia Funcional (DF), Síndrome do Intestino Irritável (SII), Migrânea Abdominal e Dor Abdominal Funcional Sem Outra especificação (HYAMS JS, et al., 2016). 
Os DFTGI têm origem multifatorial e o modelo teórico mais aceito para explicá-las é o modelo biopsicossocial, que tem como substrato neuro anatômico a conexão cérebro intestinal (DROSSMAN DA, 2016). Esse circuito comunica, via neurotransmissores, os centros cognitivos e emocionais do cérebro (Sistema Límbico, constituído pelo hipotálamo, Córtex Cingulado Anterior (CCA) e amigdala) com o TGI e vice-versa. Emoções como medo, ansiedade, estímulo doloroso, e estresse podem interferir com a fisiologia do TGI (JONES MP, et al., 2006). Assim, distúrbios de motilidade, hipersensibilidade visceral, alteração da mucosa, da microbiota intestinal e do processamento do sistema nervoso central fazem parte da fisiopatologia da dor funcional e são descritos a seguir:

\section{Hipersensibilidade visceral e alteração da modulação central da sensação de dor}

A dor tem um componente sensório- discriminativo e um componente afetivo-motivacional (KLEINMAM $\mathrm{RE}$, et al., 2018). O primeiro compreende a localização e intensidade da dor, seguindo a via do intestino em direção à medula espinhal, porção ventral posterior do Tálamo, Ínsula e Lobo Temporal. O componente afetivo-motivacional compreende o sofrimento resultante da dor e usa via medula espinhal em direção à formação reticular do tronco cerebral, via porção medial do tálamo para o sistema límbico, particularmente para o CCA, que proporciona a sensação desagradável da dor (KLEINMAM RE, et al., 2018).

A Hipersensibilidade visceral é resultado da sensibilização dos neurônios entéricos e medulares, modulação anormal das vias ascendentes, da integração cortical e das vias descendentes inibitórias (JONES MP, et al., 2006). O paciente com dor funcional tem regulação anormal do eixo Hipotálamo-Hipofisário-Adrenal (EHHA) e maior liberação de cortisol à estimulação visceral (CHANG L, et al., 2009). Como consequência, baixo limiar de dor para sensações retais ou sensação de desconforto pós prandial podem ser observados (CHANG L et al., 2009).

\section{Alteração de motilidade e de permeabilidade intestinal}

Lentificação do esvaziamento gástrico e menor amplitude de contrações do antro em pacientes com DAC funcional sugerem a participação de distúrbios de motilidade na patogênese da dor (DEVARANAYANA NM, et al., 2012).

Nos indivíduos saudáveis a barreira intestinal (constituída pelas secreções, pela microbiota e pelo epitélio) protege o organismo contra componentes do conteúdo luminal e regula a permeabilidade intestinal (SHULMAN RJ, et al., 2014). Algumas lesões podem aumentar a permeabilidade intestinal e facilitar o acesso do conteúdo luminal através da parede intestinal, provocar reações inflamatórias e modular as funções sensorial e motora (SHULMAN RJ, et al., 2014).

\section{Fatores genéticos}

A criança com DAC tem maiores chances de ter um familiar, especialmente a mãe, com um DFTGI (BUONAVOLONTÀ R, et al., 2010). Esta recorrência familiar dos DFTGI sugere uma transmissibilidade genética, no entanto, genes específicos que possam estar envolvidos de maneira a explicar a etiologia isoladamente, ainda não foram encontrados. Há evidências de que a aprendizagem de comportamento perante a doença e os fatores ambientais como dieta e estilo de vida, tenham um papel mais expressivo do que os fatores genéticos na origem da DAC funcional (BUNAVOLONTÀ R, et al., 2010, RAMCHAMDANI PG, et al., 2006).

\section{Inflamação}

A ocorrência de gastroenterite bacteriana, por exemplo, por Shigella, pode ser associada ao desenvolvimento de SII em algumas crianças (THABANE M, et al., 2010). A infecção pode levar à inflamação, alteração de microbiota e aumento da permeabilidade intestinal (SIMRÉN M, et al., 2013). Apesar da resolução da inflamação aguda, podem ocorrer mudanças persistentes no aparato neuromuscular intestinal (THABANE M, et al., 2010).

\section{Microbiota}

O subgrupo de crianças com infecção intestinal e que evolui com SII e DF sugere que a microbiota intestinal possa ter uma participação na patogênese da DFTGI (SIMRÉN M, et al., 2013). A hipótese é a de 
que mudanças na microbiota contribuam para as DFTGI através de interações com fatores do hospedeiro, como idade, dieta e constituição genética. Estas interações se relacionam a mudanças na função neuro sensorial do intestino e da função de barreira do intestino e /ou do eixo cérebro intestinal (SIMRÉN M, et al., 2013).

\section{Eventos estressantes precoces ou tardios}

O início da vida representa um período de vulnerabilidade aos fatores de estresse, devido à grande neuroplasticidade cerebral nos primeiros anos, sendo ainda, uma fase de imaturidade das vias inibitórias descendentes e dos sistemas sensoriais somáticos e viscerais (HERMANN C, et al., 2006).

Eventos estressantes, físicos e psicológicos, nas fases iniciais da infância têm sido apontados como fatores contribuintes para a sensibilização visceral aferente, com repercussões ao longo da vida (CHITKARA DK, et al.,2008; RAMCHANDANI PG, et al., 2006). É demonstrado que crianças com DAC têm mais antecedentes traumáticos como doenças, internações e cirurgias, e mais estresse associado a esses eventos do que os controles saudáveis (BONILHA S e SAPS M, 2013). Sofrer bullying ou estresse em fases mais tardias na vida também têm se associado à maior tendência a apresentar DAC funcional (FEKKES M, et al., 2006).

\section{Comorbidades psicológicas}

Estudos clínicos e epidemiológicos encontram associação entre depressão, ansiedade e os DFTGI (SAPS M, et al., 2009; CAMPO JV, et al., 2004). A ansiedade parece preceder a dor, enquanto a depressão geralmente ocorre após o início da dor (SHELBY GB, et al., 2013). A hipótese é de que a depressão, a ansiedade e os DFTGI dividiriam os mesmos fatores de risco ou se constituiriam em manifestações diferentes do mesmo problema (SAPS M et al., 2009).

Mecanismos inadequados para lidar com a dor, como isolamento social ou ainda catastrofização da dor (que consiste em imaginar a pior evolução possível) são associados à depressão e à menor qualidade de vida (KAMINSKY L, et al., 2006).

\section{Diagnóstico}

\section{Avaliação clínica e os sinais de alarme (SA)}

A apresentação clínica associada a uma história médica bem estruturada com os pais e o paciente compõem a abordagem recomendada para o diagnóstico da DAC (KAMINSKY L, et al., 2018). De particular importância é avaliar os estressores ambientais, os distúrbios sociais e psicológicos de base e o histórico alimentar (HYAMS JS, et al., 2016).

A caracterização da dor inclui localização, irradiação, severidade, tempo de evolução e sinais e sintomas associados. Segundo o Comitê de ROMA IV, o diagnóstico de DAC funcional deve ser baseado em sintomas e se após avaliação médica apropriada os sintomas não puderem ser atribuídos a outra condição médica, a origem funcional da dor é confirmada (HYAMS JS, et al., 2016).

No entanto, a exclusão de doença orgânica continua sendo um desafio para o pediatra devido à heterogeneidade dos DFTGI. A presença de determinados sinais (SA) sugere a existência de uma doença de base para a DAC (Quadro 2). Assim, a identificação dos SA diminuiria a solicitação de exames desnecessários e invasivos, ajudando o pediatra a direcionar testes diagnósticos específicos (EI-CHAMMAS K, et al., 2013, CHIOU E e NURKO S, 2011).

Os níveis de evidência e o valor preditivo de alguns SA anteriormente aceitos, têm sido questionados. É o caso do despertar noturno por dor, e das dores articulares pois estes também ocorrem em pacientes com dor funcional (EL CHAMMAS K, et al., 2013). Por outro lado, presença de anemia, hematoquesia e perda de peso tem 94\% de sensibilidade para o diagnóstico de DAC orgânica (EL CHAMMAS K, et al., 2013; REUST CE e WILLIANS, 2018).

Assim, avalição clínica deve levar em consideração que os SA devem ser colocados no contexto da história clínica e do exame físico (HYAMS JS, et al., 2016). 
Quadro 2 - Potenciais sinais de alarme em crianças com Dor Abdominal Crônica.

\begin{tabular}{|c|}
\hline História familiar de Doença inflamatória intestinal, doença celíaca ou úlcera péptica \\
\hline Dor persistente quadrante superior ou inferior direito \\
\hline Perda de sangue gastrointestinal \\
\hline Perda de peso \\
\hline Desaceleração do crescimento \\
\hline Disfagia \\
\hline Odinofagia \\
\hline Vômitos persistentes \\
\hline Diarreia noturna \\
\hline Doença perianal \\
\hline Febre inexplicada \\
\hline
\end{tabular}

Fonte: Santos EAMC, et al., 2021; adaptado de Hyams JS, et al., 2016.

\section{Exames laboratoriais}

É recomendada uma investigação limitada e baseada nos achados clínicos. Alguns autores consideram a realização de exames complementares desnecessária na ausência de SA, pelo custo financeiro, por não ajudar o diagnóstico e ainda, por prejudicar a relação médico paciente (CHIOU E e NURKO S, 2011). Outros autores, porém, justificam a solicitação de exames na ausência de SA pela falta de garantias da inexistência de problemas orgânicos nesta situação (TOLONE C, et al., 2017). Na prática, observa-se que parte considerável dos pacientes com DAC acabam sendo submetidos a exames diagnósticos, alguns deles invasivos, desnecessários e o custo financeiro final pode ser elevado (DHROOVE G, et al., 2010).

De acordo com a avaliação médica, alguns exames podem ser solicitados para ajuda diagnóstica, como hemograma, Proteína C Reativa, avaliação de urina e urocultura, exames de fezes para pesquisa de parasitas, sorologia para doença celíaca, calprotectina fecal e outros (Di LORENZO C, et al., 2005). Exames de imagem como Ultrassom e Endoscopia Digestiva Alta oferecem pouco auxílio diagnostico e devem ser solicitados apenas na presença de queixas específicas (DHROOVE G, et al., 2010).

\section{Tratamento}

Nos casos em que uma doença de base é identificada, o tratamento será especificamente direcionado para a patologia encontrada (CHIOU E e NURKO S, 2011). Com relação aos distúrbios da DAC funcional, é consenso que uma boa relação médico paciente, a abordagem psicossocial e empática com o paciente e familiares, além do reforço da benignidade do problema se associam aos melhores resultados terapêuticos (DROSSMAN, 2016; NIGHTINGALE S e SHARMA A, 2020).

Particularidades como a alta porcentagem de resposta terapêutica a placebo, estimadas em 16 a $71,4 \%$ em estudos realizados em adultos, demonstram a necessidade de estudos randomizados e placebo controlados na área (PATEL SM, et al., 2005). Enfatiza-se que as intervenções terapêuticas devem ser no sentido de reduzir o sofrimento e melhorar a qualidade de vida, visando performance escolar adequada e bom padrão de sono (BRUSAFERRO A, et al., 2018).

\section{Educação do paciente e da família}

Segundo Drossman DA (2016), a educação da família e do paciente é parte importante do tratamento. Enfatizar que a dor é real, apesar da ausência de patologia de base, e que o trato digestório está reagindo de forma exagerada aos estímulos alimentares, hormonais e ao estresse. Ainda, orientar que a resposta dos pais aos sintomas é importante para o tratamento, devendo ser de suporte e compreensão, evitando o reforço do foco na doença (BRUSAFERRO A, et al., 2018). Muitos pais relutam em aceitar o papel dos fatores psicológicos na gênese da dor, mas este reconhecimento ajuda no controle dos sintomas (WALKERS LS, et al., 2012). 


\section{Intervenções dietéticas}

Modificações dietéticas são as estratégias mais comuns no manejo da criança com DAF. É frequente os pais atribuírem aos alimentos as queixas de seus filhos, pois alguns sintomas pioram no período pós prandial, porém, causas não especificas como aumento da motilidade intestinal após ingestão também podem estar envolvidas (BRUSAFERRO A, et al., 2018).

Identificar e reduzir ou eliminar substâncias da dieta que agravem os sintomas do paciente, por meio de um diário alimentar se necessário, pode ser de ajuda no controle da dor (DROSSMAN DA, 2016). As evidências atuais, contudo, são fracas no sentido de se indicar uma dieta específica para os casos de DAC funcional (BRUSAFERRO A, et al., 2018).

Alguns autores sugerem o controle da ingestão dos chamados FODMAPS (oligossacarídeos, dissacarídeos, monossacarídeos e polióis), que conhecidamente têm efeito fermentativo, em casos selecionados (de ROEST RH, et al., 2013). Ainda, a correção da ingestão de fibras nos pacientes com constipação intestinal associada à DAC parece ser benéfica (BRUSAFERRO A, et al., 2018).

Administrar probióticos para os casos de SIl pós gastroenterite ou após o uso de antibióticos, com destaque para o Lactobacillus rhamnosus GG, tem sido recomendado (HORVATH A, et al., 2011). As evidências atuais, contudo, são fracas no sentido de se indicar uma dieta específica para os casos de DAC funcional (BRUSAFERRO A, et al., 2018)

\section{Tratamento farmacológico}

O tratamento medicamentoso visa o alívio dos sintomas nos casos não responsivos às medidas anteriormente discutidas. Em determinadas situações, como na presença de distensão pós prandial, gases intestinais, contrações intestinais ou refluxo gastroesofágico a terapia antirrefluxo ou 0 uso de antiespasmódicos poderiam ser úteis (KAMINSKY L, et al., 2006).

No entanto, medicamentos de efeitos antiespasmódicos, anti-histamínicos, antidepressivos, pró-cinéticos e outros não demonstram evidências de eficácia fortes o suficiente para recomendação de uso rotineiro para DAC de origem funcional. Orienta-se cautela à interpretação dos resultados aparentemente satisfatórios de alguns estudos clínicos, pelo caráter flutuante da dor, que apresenta períodos de melhora espontânea, além de possível efeito placebo justificando a melhora clínica (MARTIN AE, et al. 2017).

Pesquisas futuras que contribuam para a melhor compreensão da etiologia da hipersensibilidade e das alterações de motilidade poderão favorecer novas estratégias de tratamento. Assim, a capacidade de modular as funções intestinais, as respostas ao estresse neuro-hormonal, as citocinas envolvidas na inflamação e o processamento central da informação de dor, são as perspectivas futuras de intervenção (KAMINSKY L, et al., 2006).

\section{Manejo psicológico}

Identificar e reverter o estresse físico e psicológico que desempenham papel no desencadeamento, exacerbação ou manutenção da dor são medidas importantes para o controle da dor (NIGHTINGALE S e SHARMA A, 2020).

A Terapia Cognitivo Comportamental (TCC), pode ser eficaz no tratamento dos distúrbios da DAC funcional, da ansiedade e da depressão (LALOUNI M, et al., 2016). A TCC consiste em melhorar a compreensão do problema, reforçar o comportamento positivo frente à dor, ensinar as habilidades cognitivas como evitar pensamentos negativos e utilizar distrações para lidar com o sintoma (LALOUNI M, et al., 2016).

\section{Qualidade de vida e prognóstico}

Os scores de qualidade de vida das crianças e adolescentes com DAC são baixos, sendo comparáveis aos dos pacientes com doença Inflamatória intestinal (YOUSSEF N, et al., 2006). A história natural da doença ainda é pouco conhecida e parcela considerável das crianças com DAC continuarão sintomáticas na fase adulta. História familiar positiva de DAC, recusa dos pais em aceitar o papel dos problemas psicossociais na gênese da dor, excesso de exames e consultas médicas, baixa autoestima e perfil disfuncional para lidar com o problema, relacionam-se com à persistência da dor (WALKER LS, et al., 2012). 


\section{CONSIDERAÇÕES FINAIS}

A DAC em crianças e adolescentes na maioria das vezes representa um distúrbio de etiologia funcional, de fisiopatologia complexa e que requer uma abordagem biopsicossocial para evolução favorável. Uma abordagem gradual com educação, controle dos fatores de estresse e intervenções dietéticas é fortemente recomendada. Muitos estudos ainda são necessários para otimização do tratamento e compreensão da DAC funcional em pediatria.

\section{REFERÊNCIAS}

1- APLEY J, NAISH N. Recurrent abdominal pains: a field survey of 1,000 school children. Arch Dis Child, 1958;33(168):165-170.

2- BONILLA S, SAPS M. Early life events predispose the onset of childhood functional gastrointestinal disorders. Rev Gastroenterol Mex, 2013;78(2):82-89.

3- BRUSAFERRO A, et al. The Management of Paediatric Functional Abdominal Pain Disorders: Latest Evidence. Pediatr Drugs, 2018; 20:235-247.

4- BUONAVOLONTÀ R, et al. Familial aggregation in children affected by functional gastrointestinal disorders. J Pediatr Gastroenterol Nutr, 2010;50(5):500-505.

5- CAMPO JV, et al. Recurrent abdominal pain, anxiety, and depression in primary care. Pediatrics, 2004;113(4): 817824.

6- CHANG L, et al. Dysregulation of the hypothalamic-pituitary-adrenal (HPA) axis in irritable bowel syndrome. Neurogastroenterol Motil, 2009;21(2):149-159.

7- CHITKARA DK, et al. Early life risk factors that contribute to irritable bowel syndrome in adults: a systematic review. Am J Gastroenterol, 2008;103(3):765-774.

8- CHIOU E, NURKO S. Functional abdominal pain and irritable bowel syndrome in children and adolescents. Therapy, 2011;8(3):315-331.

9- De ROEST RH, et al. The low FODMAP diet improves gastrointestinal symptoms in patients with irritable bowel syndrome: a prospective study. Int J Clin Pract, 2013;67:895-903.

10-DEVANARAYANA NM, et al. Delayed gastric emptying rates and impaired antral motility in children fulfilling Rome III criteria for functional abdominal pain. Neurogastroenterol Motil, 2012;24(5):420-425.

11-DHROOVE G, et al. A million-dollar work-up for abdominal pain: is it worth it? J Pediatr Gastroenterol Nutr, 2010;51(5):579-583.

12-Di LORENZO C, et al. Chronic abdominal pain in children: a technical report of American Academy of Pediatrics and the North American Society for Pediatric Gastroenterology, Hepatology and Nutrition. J Pediatr Gastroenterol Nutr, 2005;40(3):249-261.

13-DROSSMAN DA. Functional gastrointestinal disorders: history, pathophysiology, clinical features and ROME IV. Gastroenterology, 2016; 6(150):1262-1279.

14-EL-CHAMMAS K, et al. Red flags in children with Chronic abdominal pain and Crohn's disease- a single center experience. The Journal of Pediatrics, 2013;162(4):783-787.

15-FEKKES M, et al. Do bullied children get ill, or do ill children get bullied? A prospective cohort study on the relationship between bullying and health-related symptoms. Pediatrics, 2006;117(5):1568-1574.

16-HERMANN C, et al. Long-term alteration of pain sensitivity in school-aged children with early pain experiences. Pain, 2006;125(3):278-285.

17-HORVATH A, et al. Meta-analysis: Lactobacillus rhamnosus GG for abdominal pain-related functional gastrointestinal disorders in childhood. Aliment Pharmacol Ther, 2011;33:1302-1310.

18-HYAMS JS, et al. Childhood functional gastrointestinal disorders: child/ adolescent. Gastroenterology, 2016;150(6):1456-1568.

19-JONES MP, et al. Brain-gut connections in functional Gl disorders: anatomic and physiologic relationships. Neurogastroenterol Motil, 2006;18(2):91-103.

20-KAMINSKY L, et al. Psychological correlates of depression in children with recurrent abdominal pain. J Pediatr Psychol, 2006;31(9):956-966.

21-KLEINMAM RE, et al. Walker's Pediatric Gastrointestinal disease. $6^{\text {th }}$ Ed. North Carolina. 2018, p. 2717-2778.

22-KORTERINK JJ, et al. Epidemiology of pediatric functional abdominal pain disorders: a meta-analysis. PloS One, 2015;10(5): e0126982.

23-LALOUNI M, et al. Exposure-based cognitive behavior therapy for children with abdominal pain: A Pilot trial. PLoS One, 2016;11(10):e0164647.

24-MARTIN AE, et al. Pharmacological interventions for recurrent abdominal pain in childhood. Cochrane Database Syst Rev, 2017;3(3):CD010973.

25-NIGHTINGALE S, SHARMA A. Functional gastrointestinal disorders in children: What is new? J Paediatr Child Health, 2020;56(11):1724-1730. 
26-PATEL SM, et al. The placebo effect in irritable bowel syndrome trials: a meta-analysis. Neurogastroenterol Motil, 2005;17(3):332-340.

27-RAMCHANDANI PG, et al. Early parental and child predictors of recurrent abdominal pain at school age: result of a large population-based study. J Am Acad Child Adolesc Psychiatry, 2006;45(6):729-736.

28-REUST CE, WILLIAMS A. Recurrent Abdominal Pain in Children. Am Fam Physician, 2018 ;97(12):785-793.

29-SAPS M, et al. A prospective school-based study of abdominal pain and other common somatic complaints in children. J Pediatr, 2009;154(3):322-326.

30-SHELBY GD, et al. Functional abdominal pain in childhood and long-term vulnerability to anxiety disorders. Pediatrics, 2013;132(3):475-482.

31-SHULMAN RJ, et al. Associations among gut permeability, inflammatory markers, and symptoms in patients with irritable bowel syndrome. J Gastroenterol, 2014;49(11): 1467-1476.

32-SIMRÉN M, et al. Intestinal microbiota in functional bowel disorders: a Rome foundation report. Gut, 2013;62(1):159176.

33-THABANE M, et al. An outbreak of acute bacterial gastroenteritis is associated with an increased incidence of irritable bowel syndrome in children. Am J Gastroenterol, 2010;105(4):933-939.

34-TOLONE C, et al. Recurrent abdominal pain in children underlying pathologies in absence of alarm symptoms. Minerva Pediatr, 2017; 69(4):239-244.

35-WALKER LS, et al. Functional abdominal pain patient subtypes in childhood predict functional gastrointestinal disorders with chronic pain and psychiatric comorbidities in adolescence and adulthood. Pain, 2012;153(9):1798-1806.

36-YOUSSEF N, et al. Quality of life for children with functional abdominal pain: a comparison study of patients' and parents' perceptions. Pediatrics, 2006;117(1):54-59. 